\title{
Computer Programming and Auxiliary Learning Processes: Hackers School Case
}

\author{
Adriano Canabarro Teixeira, Fernanda Batistela, Adriana Dickel \\ Universidade de Passo Fundo, Passo Fundo, Brasil \\ Email: teixeira@upf.br
}

How to cite this paper: Teixeira, A. C., Batistela, F., \& Dickel, A. (2017). Computer Programming and Auxiliary Learning Processes: Hackers School Case. Creative Education, 8 , 1001-1019. https://doi.org/10.4236/ce.2017.87073

Received: April 18, 2017

Accepted: May 30, 2017

Published: June 2, 2017

Copyright ( 2017 by authors and Scientific Research Publishing Inc. This work is licensed under the Creative Commons Attribution International License (CC BY 4.0).

http://creativecommons.org/licenses/by/4.0/

\section{c) (i) Open Access}

\begin{abstract}
In a world profoundly transformed by computer technology and connection, software assumes strategic role of human and social development. In this sense, the perception that it is fundamental for the contemporary citizen to develop software development skills gains strength and begins to popularize initiatives that go in this direction. This study aims, from theoretical and pedagogical contributions and field research, to analyze how computer programming, School Hackers Project's focus, influences the auxiliary processes of learning exposed by Juan Ignacio Pozo in his Apprentices and Masters literary work: the new culture of learning. Hackers School Project, in activity since 2014, attends elementary school students of municipal schools in Passo Fundo/RS, Brazilian medium size city. It consists of a set of actions that facilitate the learning of techniques and programming skills to elementary students through Scratch software, developed by Massachusetts Institute of Technology. This article is the result of the monitoring of project activities in one of the schools participating in the project during the year 2014. After analyzing the data collected, it was possible to verify that the factor of the greatest influence in the learning of the programming for the study group was the motivation.
\end{abstract}

\section{Keywords}

Computer Skills, Computer Programming, Educational Technology, Educational Resources, Contemporary Culture

\section{Introduction}

It is possible to verify the growing role and importance that technological devices have assumed in the human development process, whether on an individual, social, political or cultural perspective. However, it is necessary to recognize that the vector supporting these processes resides primarily in the software. That is, it is computer programs, rather than physical support, that provide true transfor- 
mations in the ways in which society and its components are organized.

In a way, our lives are intimately tied to software that controls equipment, giving us access to information, enhancing communication, putting us in contact with remotely located people, sustaining economic processes, influencing politics and, in a more complex perspective, although they are fruits of the human intellect, they begin to replace human neural processing in intellectual tasks that have some kind of predictability and repetition. In this sense, if the contemporary world depends on the software we produce, one of the fundamental elements for the exercise of citizenship is the development of software development competences. In the context of this analysis, we also point out that developing software helps directly in the development of cognitive skills that have consequences in the life school of individuals. So, it is believed that programming computer is part of the basic skills of the citizen of the 21 st century.

Among the numerous initiatives around the world to teach programming for children and adolescents, one is held in Passo Fundo, a medium-sized city in the state of Rio Grande do Sul, Brazil. Denominated School of Hackers attends the public schools of the municipality and, since 2014, already formed more than 700 children. The project consists of a set of actions that allow the learning of programming techniques and skills through the software Scratch, to students from the 6th to the 9th year of Elementary School and to teachers of municipal schools of Elementary Education in Passo Fundo. In 2015, the project was awarded with Rio Grande do Sul State Legislative Assembly Leaders \& Winners Award.

The aim of this study was to analyze how computer programming, a focus of the School of Hackers Project, influences the auxiliary learning processes presented by Juan Ignacio Pozo in his work Apprentice and Master: the new culture of learning. Held during the first edition of the Project in 2014, it was guided by four stages: Preliminary stage-definition of contents and methodology to be used by the monitors in the enrolled schools; Stage of execution-realization of workshops; Graduation and Evaluation of the project. We chose the programming environment Scratch to develop the activities of the School of Hackers because this resource was already being used in the Computers Programming Games for Elementary Education, from which originated the School of Hackers project. In 2014, the Project had the total participation of twenty-one teams, summing a number of 312 students from Elementary School for six monitors.

As methodological procedures, the study took place between February and December and had data sources of the following resources: bibliographic research, interviews with the research subjects, monthly questionnaires, reading the students' records; participation in meetings with the organized committee of the project; classroom memories of the monitors and observation with a class of eleven students-six girls and five boys, from one of the participating schools.

The selection of students was carried out by the school management team and ranged from 15 to 20 participants per class, who met in two hours a week in the computer lab of the schools, from May to December, in the reverse shift to 
school activities. The school chosen for this study was Notre Dame, because it is the pilot school.

The students participating in the School of Hackers dedicate themselves to the Project workshops because they have their own motivation, once they are not obliged to be present, they do not receive a note, concept or score for the challenges that are programmed and are not approved or disapproved, and they only program. The School of Hackers is part of a context characterized by Juan Ignacio Pozo, who says that we live today the knowledge society, where the demands of learning lead citizens to learn "more and more things, but to learn in another way, within of a new learning culture" (Pozo, 2004).

The motivation, attention, recovery and transference of the representations present in the memory of the subjects and the conscience, according to Pozo, are mobilized as auxiliary processes of the learning (Pozo, 2002), which in the Hackers School context, is believed to be influenced by the possibility of programming, through the interventions of the monitors, who have experience on the Scratch environment and the individuals who are participating in this learning process. This mobilization of the cognitive system, according to the author, is essential for the individual to learn. In this sense, the problem of this research is constructed as follows: To what extent does the programming of computers, the focus of the Project School of Hackers, activate the auxiliary processes of learning proposed by Juan Ignacio Pozo?

The research is qualitative and exploratory, and the theoretical matrix from which the categories of analysis were originated, comes from the studies on the four auxiliary processes of learning - motivation, attention, recovery and transference and the consciousness-Juan Ignacio Pozo in his Apprentices and masters: the new culture of learning, dismembered into twelve subcategories, which are the three main forms of manifestation of categories, in order: for reasons, expectation, intervention; by control, selection and surveillance; by recognition, evocation and transfer; planning, regulation and evaluation.

Of these, it was observed that the data sources mentioned above, documented through research in the field, were analyzed 1065 incidents, with 466 reported the motivation as the most influential factor in learning programming, enabled by internal reasons (intrinsic) and/or external (extrinsic). In addition to this process, the consciousness was evidenced through the application of three types of control: task planning, regulation and evaluation of the own productions and results. Finally, and peripherally, the question of research was identified that the eleven students, whose link to the Project that was analyzed, constructed knowledge about computer programming that extrapolated those coming from the monitors.

\section{Systematizing the Four Auxiliary Learning Processes in Juan Ignacio Pozo}

Motivation, attention, recovery and transference and awareness are identified as auxiliary processes of learning that, in the context of School of Hackers, are ac- 
tivated by the possibility of programming, in the presence of the monitors, which have experience on the Scratch environment and the individuals who are participating in this learning process. This mobilization of the cognitive system, according to Pozo, is essential for the individual to learn.

Juan Ignacio Pozo has published many documents, articles and books, which are mostly directly related to learning, such as Apprentice and Masters: the new culture of learning (2002), for example. In translation, apprentices and masters mean students and teachers, and Pozo uses this sphere to write about learning as a psychological process in a context not only linked to the school, but in a general context where you teach and where you learn. Pozo structures his work in four parts, according to Figure 1, and in the second, presents a model of the human cognitive system and the main processes that contribute to learning, in which the presence of the four auxiliary processes of learning will be emphasized.

To understand the processes related to learning activities, it is necessary to situate them, first, in the social context in which they were generated, in their history. In the past, learning was reproductive and students wrote and rewrote to memorize such content to reproduce it easily. This time cannot be compared to the demands of the new culture of learning, both in quality and in quantity. This new culture, often mentioned by Pozo in the course of his work, makes the human being learn more and more things. Pozo says that "there was never a time when so many people learned so many different things at the same time, and so many people dedicated to make other people learn. 'We are in the learning society'." (Pozo, 2002).

The author points out that, possibly, the human being is producing learning, to a greater or lesser degree, in all his activities or behaviors. One of the theses that Pozo develops is that every learning situation will always imply different types of results, processes and conditions. For this, it is necessary to accept that it is endowed with interconnected memory systems, so in this new society, composed of a whirlwind of information, the memory of the human being ends up rebuilding the information that receives, rather than recording them, reproduce them or serve as a cultural archive in the traditional sense. Memory is one of the components that integrate the cognitive processes of psychology. These,

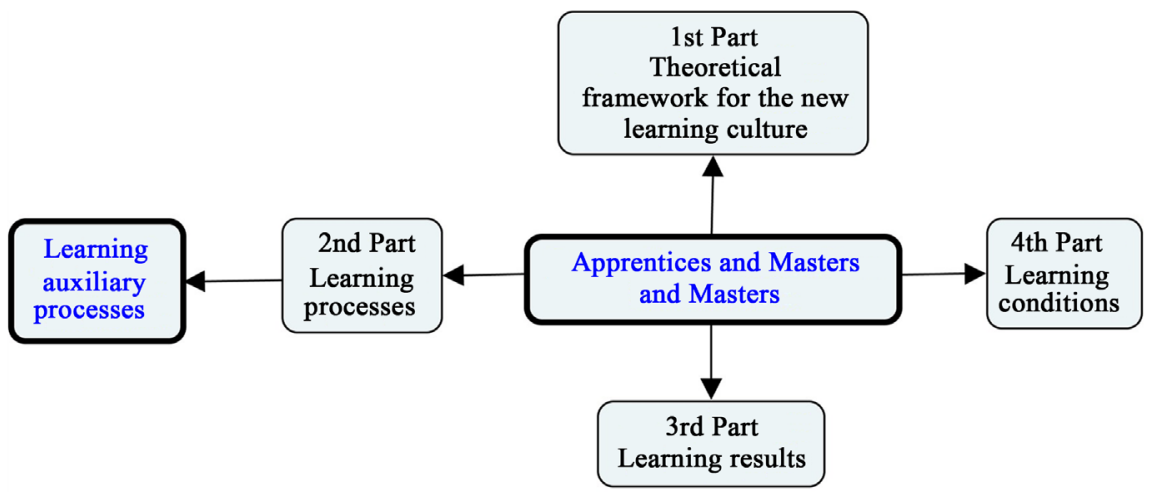

Figure 1. Structure of the work of Juan Ignácio Pozo. 
integrated with a combination of other auxiliary processes, influence in different ways and in different learning situations, depending on their objectives and conditions.

With the intention of expand the understanding of the learning processes that occur in humans, especially among eleven students participating in the School of Hackers project, in a new school context, with the use of computer programming, it was decided to highlight the four auxiliary processes of learning - motivation, attention, recovery and transference, and consciousness, to serve as base of research field data analysis. It is worth mentioning that each category has its own characteristics, but at the same time, they complement each other. Together, they provide the individual with greater learning, which reflects a greater awareness of its own cognitive functioning. In this way, each of them will be treated quickly, beyond the forms of manifestation, which are shown in Figure 2 and are in the follow order: for reasons, expectation, intervention; By control, selection and surveillance; By recognition, evocation and transfer; Planning, regulation and evaluation.

Motivation is the first auxiliary process of learning, listed by Pozo, and its main forms of manifestation are through motives, expectations and intervention. According to Pozo (2002), learning implies to have motivation and the human being must have reasons to learn, either by the extrinsic or intrinsic form of manifestation.

The first form occurs when the "learning motive is not what you learn, but the consequences of having learned it" (Pozo, 2002: p. 139), that is, it is perceived when the individual focuses on the consequences of his learning, and not in the

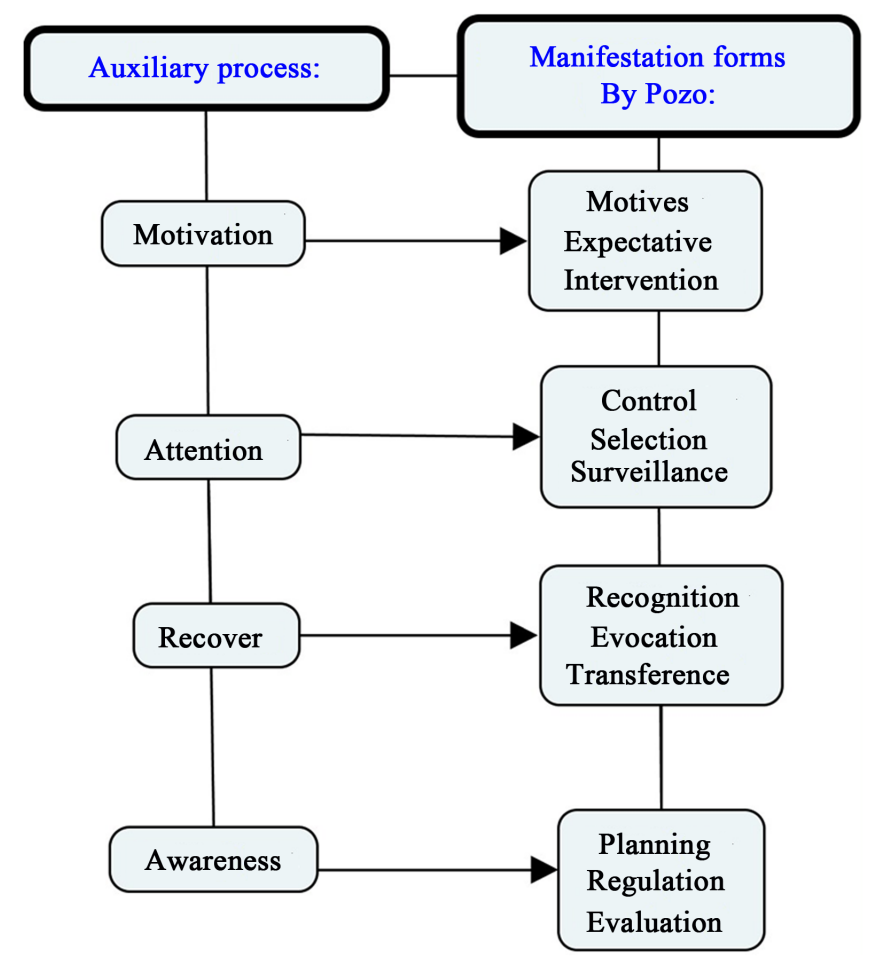

Figure 2. Category analysis. 
activity of learning itself, because it wants to achieve something desired or wants to avoid something undesirable, such as a promotion at work. To do that, you will study English to increase your salary, for example. Therefore, the motive for this motivation is outside of what you learn. On the other hand, "the fact that students perceive that a learning result is significant or has an interest in itself is another reason to learn, which is known as intrinsic motivation" (Pozo, 2002: p. 140 , author's Griffin), that is, it requires internal motives to learn and the individual is motivated to learn something by the desire to learn, for example, to learn English by the desire to speak this language.

In addition, motivation also depends on the expectation of success that the individual expects or tries to achieve. Thus, by realizing that your expectations are not being met, your motivation decreases and you may no longer strive to achieve that goal. So, in addition to valuing the perceived progress in the learning of individuals, there are two ways to increase motivation and collaborate for learning, which is to increase the expectations and value of this expected success. The form of manifestation of motivation by expectation means that, "if a result does not interest us, we will not strive to achieve it. But even if we are interested, we will try very little if we think that we will not reach it or that we do not know how to do it because we lack habits [...] to achieve it" (Pozo, 2002: 142).

Finally, teachers can give reasons for learning by raising student expectations through good planning, for example, tailoring tasks to each student's capabilities and connecting activities to their interests. Also, it is emphasized that the way the teacher will face the task of teaching is that he will move or not, the students to the learning and the motivation that the teacher manifests will not be disconnected from the motivation of the students themselves, because they see it as a mirror. Thus, teachers' attitude may reflect on students attitudes and learnings, since "no one will lead others to learn if there is not a learning movement" (Pozo, 2002: p. 145). In this way, it is possible to intervene in the motivation of the individuals and, the more valued they feel, the easier it is to internalize this success and to recognize it, directly influencing their learning.

Furthermore being treated of manifestation by intervention, Pozo relates the motivation also to the situations in which the individuals need to solve, which are identified as problems. "To perceive something as a problem requires an apprentice 'motivation' for the task, beyond the rewards he receives for doing it well" (Pozo, 2002: p. 254, author's Griffin), that is, not always the problems which are considered by teachers are also perceived as a problem of interest to students. In such a way, the more new and unpredictable tasks are, the greater the tendency of students to perceive as real problems to be solved than a mere exercise.

So, Pozo presents Ten Commandments of learning, represented in Figure 3, which refer to the four auxiliary processes of learning. It is called attention to the ninth commandment, which states that proposing open tasks, such as learning problems through computer programming, promotes the cooperation of apprentices in their resolution, as "it promotes the emergence of conflicting alter- 


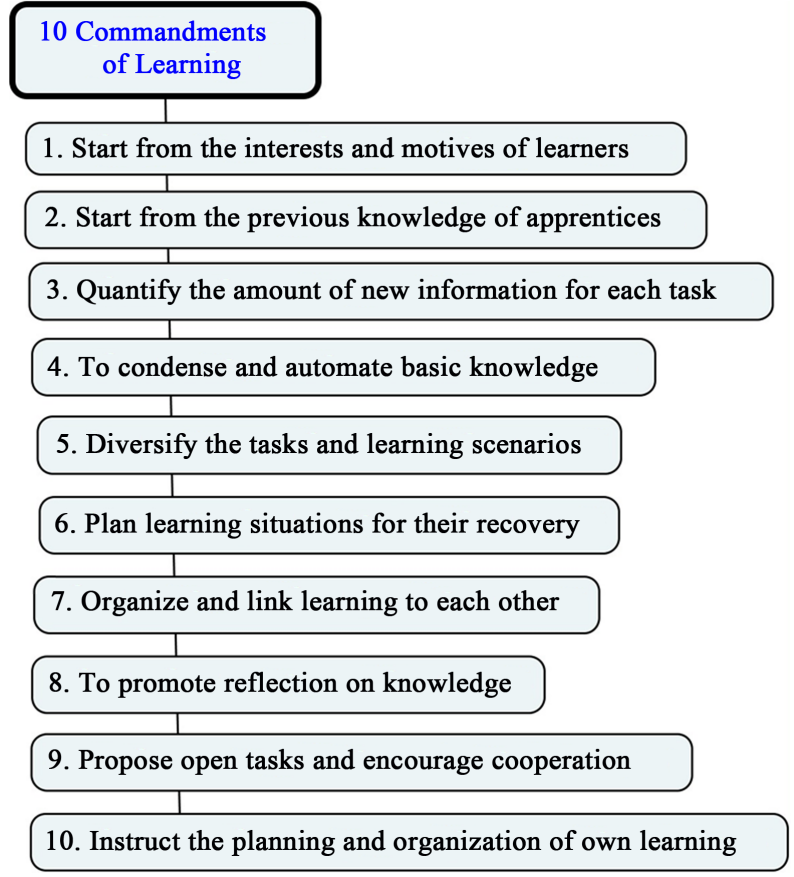

Figure 3. The ten commandments of learning.

natives as well as mutual aid between students in order to achieve the common goals proposed [...] and to promote motivation for their own learning" (Pozo, 2002: p. 272).

Briefly, learning implies having motivation, which can be for reasons, for expectation or for intervention. The first is perceived in the extrinsic form, when the individual focuses on the consequences of his learning and not on the activity of learning itself, and intrinsically requires internal motives to learn, that is, the individual is motivated to learn something by the desire to learn. In the second, "motivation does not depend only on the motives we have, but success that we expect if we try to reach them" (Pozo, 2002: p. 142). The third is when the teacher intervenes in the motivation of the student, giving reasons to learn, through good planning of the class. In addition, Pozo (2002) says that motivation alone does not guarantee learning. It is need to activate other processes, such as attention, which is linked to working memory.

Attention is the second auxiliary process of learning and its main forms of manifestation are the three mechanisms or, also identified by Pozo as a function of the human attention system: the control of limited resources, the selection of the information that must be processed and the surveillance or alert, which allows sustaining attention. "The three functions of the attention system (control, selection, and surveillance) can become more effective in learning if we teachers help students follow certain principles." (Pozo, 2002: p. 150).

This learning process is closely linked to working memory, which needs to be activated so that the individual can process the most relevant information and remind them in the future. Even so, this does not always happen, precisely because the individual has a limited memory. This is due to several causes or me- 
chanisms, which can be manifested through control, selection and surveillance.

The first, relates to a control system that is limited, that is, attention works like gasoline as it is used is also consumed. According to Pozo (2002: p. 147), "when a task, like read this book, requires that we use controlled processes, concentrating our attention on it, we have scarce resources for other subsidiary tasks" and automated when errors are not detected in a given action, therefore, it acts without attention.

The second mechanism concerns the selection of information that must be processed. This is because "we tend to pay more attention to interesting information, which has to do with our motivation" (Pozo, 2002: p. 148), at the moment.

The third mechanism refers to vigilance, which allows us to sustain attention for a continuous time, even though, a time will come when its resources are depleted (Pozo, 2002). Therefore, it is necessary to recover forces and better distribute the resources. It is important that the individual has the autonomy to organize the learning place, so that he distributes his resources strategically. To facilitate this process of activation of the attentional system in the school context, there are some mechanisms, such as selecting the most relevant information or helping the student select, dosing and diversifying the tasks, as well as presenting them in an interesting way. With this, they can obtain a greater attention under a certain knowledge and, therefore, a greater learning.

After attention, the next auxiliary process of learning is recovery. Its main forms of manifestation are by recognition, evocation and transference of learning to new contexts. This process is perceived at the moment on what the individual recovers, in some way, a learning that has already happened. Thus, he can recover in a more difficult way, through evocation, when he has many variables and few indications that refer to learning to be recovered and, through recognition, when he receives a stimulus with several clues and little or no variable, that is, "the less evidence there is, the more difficult recovery will be" (Pozo, 2002: p. 153). This stimulus retrieves information that brings with it much other information regarding desired learning, facilitating the recovery.

Pozo says that recognition precedes evocation and provides an example to explain the two processes.

"[...]. Sometimes [sic], in the crowded supermarket aisles, we collide with another cart that is casually being driven by a person whom we immediately recognize as a former co-worker. The presence of a stimulus or an estimate configuration (The goatee and the turtle-rimmed glasses) makes us recover an acquired representation of that stimulus, which brings with it attached or connected other information (after all, did you set up the office? What happened with Helena, your girlfriend at the time? Do you know anything about Raul, yes, the one that stuck on all the exams?)". (Pozo, 2002: p. 152, author's Griffin).

We conclude that the greater the evidence and the smaller the variables, the easier it will be to recover an apprenticeship. Still, it is worth emphasizing that the greater the individual's awareness of his/her own knowledge, the frequency 
of use of learning, and the more varied the contexts in which such knowledge was learned, and the more similar the contexts of learning and recovery, easier it will be the recovery and transfer. Thus, "transference is one of the central characteristics of good learning and, therefore, one of its most usual problems" (Pozo, 2002: p. 63). Finally, when the individual learns to use the same knowledge in different situations, the easier it will be to transfer the same to different contexts and, therefore, will have a more lasting learning.

Briefly, awareness can help students better target their learning when they can plan, regulate, and evaluate the results. In addition, the author encourages these practices to be carried out ever more distant from the teacher's help. Consciousness is the fourth and last auxiliary process of learning and its main forms of manifestation are through three types of control mentioned by Pozo (2002): task planning, regulation of its execution and evaluation of its results.

The first refers to routine situations, in which the human being does not set goals to put them into practice and non-routine situations, in which, when encountering problems, the individual ends up elaborating a strategic plan to guide their activities. This planning of a learning task "implies setting before the beginning the goals and the means to reach it” (Pozo, 2002: p. 160).

Concomitantly, the regulation comes with planning, that is, in addition to these established goals, sub-targets are drawn, which will serve to regulate the practice itself and correct any possible errors or deviations. From this, it is possible to detect inconsistencies that, in time, can be corrected, guaranteeing a better strategy. According to Pozo (2002: p. 160, author's Griffin), the sub-goals are "small 'landmarks' that indicate that we are going in the right direction."

Finally, the third concerns the evaluation of the results achieved, at which time the individual will reflect on their ways of learning, according to the goals and sub-goals outlined. In addition, you can assess the inconsistencies of your strategies, that is, where your conscience has done certain things, using automated and controlled processes, since the human being can only allocate his/her cognitive resources to a part of the stimuli. In this way, the individual makes an evaluation on the own productions; makes a self-assessment, which "is an essential component of self-control in all kinds of learning tasks" (Pozo, 2002: p. 161).

Once this synthesis of the auxiliary processes of learning is made, it is fundamental to briefly explore the social context on which the research focuses on the role of educational informatics, the profile of the student of the 21st century and on the consequences of the act of programming in yes. These elements were decisive for the answer to the research question.

\section{Computer Programming: The Relation between Educational Computing, the Profile of the 21st Century Student and Auxiliary Processes of Learning}

Juan Ignacio Pozo, in his works, makes it clear that there has never been in the history of mankind a society composed of a large number of people learning in such proportions and concomitantly. Therefore, he identifies it as a learning so- 
ciety, because it requires that the human being is always in search of learning more things and in different ways. One of these ways of learning is through computer science, which in itself is educational and the contemporary social context points to a tendency of non-existence of education without computer science.

Starting from the point of view of its contextualization, the computer science, more specifically, the computer, according to Valente, arose with the primordial intention to solve the problems in courses of postgraduate and like teaching machine, from its capacity of programming. Making this correlation between the emergence of the computer in 1958 and the time in which this study is being carried out, fifty-seven years have passed. Thus, it is concluded that the purpose of the machine has not changed and this idea is defended by many scholars of the area, which show that everyone should program, otherwise people will be programmed by the machine, as Rushkoff (Rushkoff, 2012).

Papert, in his experience with the LOGO programming language, asserts that individuals create, think, invent, experiment, use the cybernetic animal identified by a tortoise to move it according to the command itself, which makes them act in a autonomy manner, to plan, regulate and evaluate their own constructions of knowledge (Papert, 1985).

Douglas Rushkoff, in his book The 10 Essential Issues of the Digital Age, Program your future not to be programmed by its, emphasizes the importance of computer programming, especially in the 21 st century, which is considered different from previous ones, being of digital era, where programming is present and "means to determine the codes and rules by which our many ICTs will build the future." (Rushkoff, 2012). This is, the individual who was born in this age possesses the skills of the student of the 21 st century and has the autonomy to create his own rules, which, according to the author, will attribute future reflections to his life in the world, if this does not happen, then the Human being will be bound to the limitations of the computer and will be at the mercy of being programmed by it.

Pozo says that it is important to form students endowed with learning abilities, capable of building their particular truths among so many partial truths, and that they are prepared to use this information strategically and assimilate it critically. According to the author, using information strategically consists of converting the information into an ordered knowledge and true knowledge. Still, in the information society in which we live, few can access this ability to sort information by transforming it into knowledge (Pozo, 2004).

Bonilla complements Pozo's statement, saying that when the individual feels challenged, he ends up seeking, without the help of other people, the best ways to create, make and propose tasks, which influences the transformation of information into knowledge (Bonilla, 2005). Still, this process is part of the first mechanism or function of the attentional system, related by Pozo, which divides it into controlled, attentive and automated processes without attention. In this 
perspective, Pozo says that when the individual can do things without the help of other people, it is because he does not need "crutches" anymore (Pozo, 2002). It compares this learning process to the construction of a work, firstly, the teachers put "scaffolding" (Pozo, 2002), that is, they lend their conscience to the student, and gradually remove these scaffolding, so that the student can have selfawareness and no longer need crutches. Pozo also explains that when the student no longer needs these scaffolds or crutches, it is because he has become aware of his own cognitive functioning (Pozo, 2002). Therefore, he can plan his tasks, regulate his own execution activity and reflect on his productions, that is, evaluate the results of his own productions.

When programming, the individual will be continually searching for appropriate commands to the situation he wants to program, so it is necessary to activate the creative and autonomous capacity, because he will constantly test his learning and will not have his answers as right or wrong but resolved, as he was able to make the programming happen as he wished. So, the individual who programs will always be seeking answers and, gradually, will be programming autonomously, without needing help. Over time, he will be able to make his choices and therefore be more aware of his own learning and of his role in the world.

The educational interest of computer programming runs not only from its economic importance, but also from its value as a learning environment. Computer programming can be explored to develop skills such as problem solving, creative thinking, logical reasoning, systematic experimentation and, recently as a form of literacy, for a modern society, where programming is embedded as a compulsory school subject, because it allows citizens to become active producers of interactive digital content for web, facilitating the learning process (Fesakis \& Serafeim, 2009).

We conclude that computer programming is entirely linked to learning and who program becomes a producer of software, educational objects, learning objects. According to Papert, programming software favors learning, since it gives autonomy to the student and says that "the best learning occurs when the learner assumes the command" (Papert, 1994), that is, when he has control over learning itself. For this, necessarily the individual will enjoy the four auxiliary processes of learning and their forms of manifestation.

In video Scratch, Media Lab Video, Mitchel Resnick and other researchers from the MIT Laboratory explain about their goals in creating Scratch and its potentialities. They say that with Scratch, the programmer will have control over their projects, deciding what changes they want to make. Jay Silver says he likes "the idea of a tool that can be used in schools, but that induces the learner to use it alone" (Resnick, 2007: p. 1-38) and that one of their goals is to create a worldwide community of creators to share their projects with friends from all over the globe, as they will learn from each other.

Scratch, whose slogan is imagine, program, sharing, is a programming system 
developed by the Massachusetts Institute of Technology (MIT), experienced in the development of educational tools for school-age children and the KIDS group at the University of California, Los Angeles. It was founded by Mitchel Resnick, director at MIT of a group called Lifelong Kindergarten, translated to Kindergarten throughout life. Its main objective in the Institute is to develop ICTs to awaken creativity in the human being, especially in children, because he believes that, as the role and the brush already aided in the learning process, now the technology comes to help as well, as it already has helped.

In this way, when the student creates something that is significant, he builds the knowledge that, according to Pozo, has to do with control over learning, because he became aware of his own cognitive functioning and ends up distancing himself more and more from the limit of give a meaning to the information he receives from the teacher Pozo (2002). But for this, the student must have a motif, which may be due to an extrinsic or intrinsic motivation; by expectation of success or failure or by intervention.

Thus, the presence of computer science, by itself, already wakes up in the individual of the 21 st century the first process listed by Juan Ignacio Pozo, which is the motivation, which stands out intrinsically, that is, the subject learns by internal reasons, that depart from the desire to learn by the personal satisfaction of being understanding or dominating an Information and Communication Technology related to educational computing. With this approach, it follows the methodology used in this study, which will show that this category of analysis, the motivation, overcame among the others.

\section{Search Method}

This is a qualitative research and the technical procedures used were, for the most part, books and the instruments of data collection were interviews, questionnaires and observation. The main universe of the researcher was the observation of a class of eleven students in grades 8th and 9th, fromElementary School at Passo Fundo School, participants of the Hacker School Project.

The study to be presented turned especially to the four auxiliary processes of learning, motivation, attention, recovery and awareness, which are depth by Pozo (2002), which were listed as four categories of analysis, which were broken down into twelve subcategories, by reasons, expectation, intervention; By control, selection and surveillance; By recognition, evocation and transfer; by planning, regulation and evaluation.

Based on the four categories of analysis, disaggregated into twelve subcategories, the data collected from the following sources were analyzed: observations of a class of eleven students; Participation of the meetings of the project team; Reading the student registration forms; Follow-up of the classroom memories of the monitor(s); Interview with the management team, with the coordinator of the computer lab, with Portuguese and Mathematics teachers, and with the attending students; Monthly questionnaire, sent to the management team, coordinator and monitor of the School and observation of the participating group of 
the Computer Programming Olympiad.

The data collected in folders created in Google Drive was recorded and distributed to documents specific to the twelve forms of manifestation. For this, a questioned Table was elaborated, available in https://goo.gl/quGGMy and represented in Table 1; Composed of the four categories, its twelve forms of manifestation and questions drawn from the work of Pozo (2002), in order to facilitate the collected data organization and, finally, to reach the final result of this study.

Each questioning was carefully elaborated, based on the citations organized for each of the manifestations. For example, when a manifestation or fact fit the question, "Is the student learning for Intrinsic reasons, that is, for the satisfaction of learning, and is the student waiting for himself, expectation of success or failure?", Was classified in motives and expectation subcategories. Done this, such manifestation was copied on online links of these two subcategories. Subsequently, it was added to the number of times they were perceived. Thus, in the same way, it was performed in each of the twelve sources of data collected in field research, totaling 1065 perceptions, which resulted in an Analysis Control Table, available in https://goo.gl/quGGMy and represented in Table 2, which included the four categories of analysis, the twelve forms of manifestation, the distribution of the data collected in the field research and the sum of all these values. The main objective of this study was to verify the number of manifestations of each of the auxiliary learning processes and to analyze if any of them would stand out in the experience.

Therefore, the study was conducted in this way: the development of the School of Hackers Project was monitored to understand its operation, especially at Notre Dame School, through the observation of a group of eleven students from the 8th and 9th years of elementary School in the course of the weekly workshops developed during seven months,fromJuintoDecember, in the reverse shift ofstudents' schoolactivities, as well as the collection of data from the other moments specified previously; a monthly questionnaire was sent to the director, the laboratory coordinator and, the workshop monitor, to check if there was any reflectionon the learning and behavior of the participating students: did they

Table 1. Part of the questions for data distribution.

Category Manifestation form
$\begin{gathered}\text { Motives } \\ \text { Is the student learning for intrinsic reasons, that is, for the } \\ \text { satisfaction of learning? Is the student learning for extrinsic } \\ \text { reasons, that is, to avoid something unwanted or to } \\ \text { achieve something desired in exchange for learning? } \\ \text { Is the student waiting for himself, expectation of success } \\ \text { or failure? Do the others expect this student to succeed or } \\ \text { fail? Is the student struggling to achieve a result? }\end{gathered}$
Intervention
Thinking about the six principles pointed out by Pozo, are the
monitors intervening in some way in the students' motivation?
Control


Table 2. Control of analyzes.

\begin{tabular}{|c|c|c|c|c|c|c|c|c|c|c|c|c|c|c|}
\hline 1 & \multicolumn{14}{|c|}{ Short link to table access: https://goo.gl/jF4H8A } \\
\hline 2 & \multicolumn{14}{|c|}{ CONTROL ANALYSIS } \\
\hline 3 & First Column & \multicolumn{3}{|c|}{ Second Column } & \multicolumn{3}{|c|}{ Third Column } & \multicolumn{3}{|c|}{ Fourth Column } & \multicolumn{3}{|c|}{ Fifth Column } & Six Column \\
\hline 4 & Collected data & \multicolumn{3}{|c|}{ MOTIVATION } & \multicolumn{3}{|c|}{ ATTENTION } & \multicolumn{3}{|c|}{ RECOVERY } & \multicolumn{3}{|c|}{ CONSCIENCE } & \multirow[t]{2}{*}{ Sum } \\
\hline 5 & 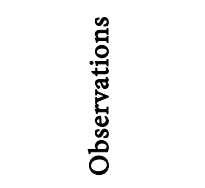 & 芯 & 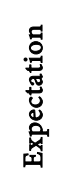 & 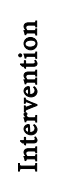 & $\begin{array}{l}\text { 몸 } \\
\text { : }\end{array}$ & 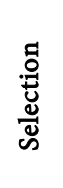 & 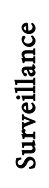 & 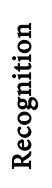 & 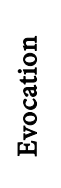 & 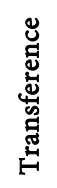 & 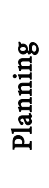 & 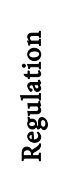 & 苛 & \\
\hline 6 & $28 / 05 / 2014$ & 3 & 4 & 1 & 4 & 4 & 0 & 1 & 0 & 2 & 1 & 0 & 0 & 20 \\
\hline 7 & $04 / 06 / 2014$ & 9 & 8 & 3 & 10 & 10 & 0 & 4 & 0 & 4 & 6 & 4 & 4 & 62 \\
\hline 8 & $11 / 06 / 2014$ & 7 & 7 & 0 & 3 & 4 & 0 & 2 & 0 & 2 & 3 & 3 & 3 & 34 \\
\hline 9 & $18 / 06 / 2014$ & 6 & 9 & 4 & 5 & 1 & 0 & 2 & 1 & 3 & 2 & 2 & 3 & 38 \\
\hline 10 & $25 / 06 / 2014$ & 6 & 8 & 3 & 4 & 2 & 0 & 3 & 1 & 2 & 2 & 2 & 2 & 35 \\
\hline 11 & 09/07/2014 & 6 & 6 & 7 & 7 & 2 & 1 & 3 & 2 & 4 & 3 & 2 & 3 & 46 \\
\hline 12 & $16 / 07 / 2014$ & 5 & 5 & 0 & 2 & 2 & 1 & 1 & 1 & 1 & 1 & 1 & 1 & 21 \\
\hline 13 & $20 / 08 / 2014$ & 10 & 9 & 5 & 1 & 2 & 0 & 3 & 0 & 2 & 1 & 1 & 1 & 35 \\
\hline 14 & $27 / 08 / 2014$ & 12 & 13 & 2 & 7 & 0 & 0 & 6 & 0 & 5 & 1 & 3 & 4 & 53 \\
\hline 15 & $03 / 09 / 2014$ & 5 & 5 & 1 & 5 & 6 & 5 & 3 & 2 & 1 & 3 & 2 & 2 & 40 \\
\hline 16 & $17 / 09 / 2014$ & 5 & 4 & 0 & 2 & 4 & 1 & 1 & 0 & 1 & 2 & 0 & 0 & 20 \\
\hline 17 & $22 / 10 / 2014$ & 9 & 10 & 3 & 10 & 11 & 3 & 4 & 3 & 3 & 3 & 2 & 3 & 64 \\
\hline 18 & $29 / 10 / 2014$ & 6 & 6 & 6 & 3 & 2 & 1 & 1 & 1 & 1 & 2 & 2 & 1 & 32 \\
\hline 19 & $12 / 11 / 2014$ & 2 & 2 & 0 & 3 & 3 & 1 & 1 & 0 & 1 & 2 & 1 & 1 & 17 \\
\hline 20 & $19 / 11 / 2014$ & 4 & 4 & 1 & 5 & 5 & 2 & 2 & 0 & 1 & 1 & 1 & 1 & 27 \\
\hline 21 & $26 / 11 / 2014$ & 6 & 6 & 6 & 5 & 5 & 0 & 3 & 0 & 3 & 2 & 0 & 0 & 36 \\
\hline 22 & $02 / 12 / 2014$ & 4 & 4 & 3 & 3 & 3 & 2 & 2 & 0 & 1 & 3 & 0 & 0 & 25 \\
\hline 23 & Interviews & 56 & 91 & 4 & 35 & 24 & 3 & 25 & 9 & 22 & 8 & 16 & 28 & 321 \\
\hline 24 & Questionnaires & 7 & 7 & 0 & 6 & 6 & 0 & 3 & 0 & 4 & 3 & 3 & 0 & 39 \\
\hline 25 & $\begin{array}{c}\text { Perceptions } \\
\text { next to the } \\
\text { monitor group }\end{array}$ & 13 & 20 & 8 & 10 & 15 & 1 & 9 & 0 & 9 & 6 & 5 & 4 & 100 \\
\hline 26 & \multirow[t]{2}{*}{ SUM } & 181 & 228 & 57 & 130 & 111 & 21 & 79 & 20 & 72 & 55 & 50 & 61 & \multirow[t]{2}{*}{1065} \\
\hline 27 & & & 466 & & & 262 & & & 171 & & & 166 & & \\
\hline 28 & Observ & $\begin{array}{l}\text { ions o } \\
\text { vith th }\end{array}$ & $\begin{array}{l}\text { Irred } \\
\text { tervie }\end{array}$ & $\begin{array}{l}\text { ain } 7 \\
\text { with }\end{array}$ & $\begin{array}{l}\text { nths. ' } \\
21 \mathrm{D}\end{array}$ & $\begin{array}{l}\text { Wed } \\
\text { tors a }\end{array}$ & $\begin{array}{l}\text { days } \\
21\end{array}$ & $\begin{array}{l}\text { wer } \\
\text { dinat }\end{array}$ & $\begin{array}{l}\text { obs } \\
\text { of the }\end{array}$ & $\begin{array}{l}\text { ed are } \\
\text { hools }\end{array}$ & 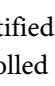 & $\begin{array}{l}\text { cause } \\
\text { he } P r\end{array}$ & $\begin{array}{l}\text { yco } \\
\text { t. }\end{array}$ & \\
\hline
\end{tabular}

perceive any change in the learning and behavior of the students participating in the Project?; an interview was conducted with the director, the laboratory coordinator and, the workshop supervisor at the beginning and end of the Project to find out if there was progress in the students' learning and behavior; at 
the end of the Project, with the eleven students of Notre Dame School, because it is a technique of data collection that facilitates interventions and that provides conditionst or each the results objectified by this research. For the students questioned about the evolution sperceived by them, since they began to participate until the graduation (at the end of the Project): what they learned? What have they improved? Did they see changes in the school context, in the disciplines and/or behavior? And in day-to-daylife, in their houses especially?; as well as the collection of data from the other moments previously specified.

Still, in the course of the research, the concepts of informatics and learning in Pozo were approached, confronting them with the characteristics of the student of today; Theoretical and practical knowledge about the Scratch programming environment and its benefits; We explored the four auxiliary processes of learning from the work of Juan Ignacio Pozo, Apprentice and Masters: the new culture of learning, and in the following topic, the students' performance will be analyzed and identified within the School of Hackers, which of these auxiliary learning processes were activated, emphasizing the process of greater number of manifestations.

It should be noted that, in the link of the Analysis Control Table, clicking on each of the manifestations, distributed between the second, third, fourth and fifth columns, will be directed to another page, which includes all the items absorbed Field research. Also, clicking on each of the dates of the observations, located in the first column, to the left of the Analysis Control Table, will be directed to the descriptions of the class memories recorded by the researcher in the observations of the workshops held in the computer lab Of the Notre Dame School from May to December 2014. The same goes for the interviews, questionnaires and perceptions with the group of monitors, which are mentioned in lines 23, 24 and 25 of the Table, just below the dates of the observation dates, in the first column.

In this way, the categories of analysis will be located in a universe of cognitive processes of learning and, with this, we will arrive at the results of the motivation of this research, which is to analyze the way in which computer programming, Project School of Hackers, influences the auxiliary processes of learning exposed by Juan Ignacio Pozo in his work Apprentice and Masters: the new culture of learning. It is important to emphasize that greater emphasis will be placed on the learning process Pozo (2002) with a greater number of incidents.

\section{Analysis of Data Collected and Results}

The Analysis Control Table illustrates the results obtained from the collection of data from the field research concomitant to the four auxiliary learning processes and their forms of manifestations, through Pozo (2002). Thus, the motivation was the one that most showed manifestations, with 466 perceptions and in second placement, attention, with 262 perceptions, remaining the recovery, with 171 perceptions and the conscience had the lowest number of perceptions, totaling 166 perceptions, according to Graph 1 . Detailed analysis of each of the 


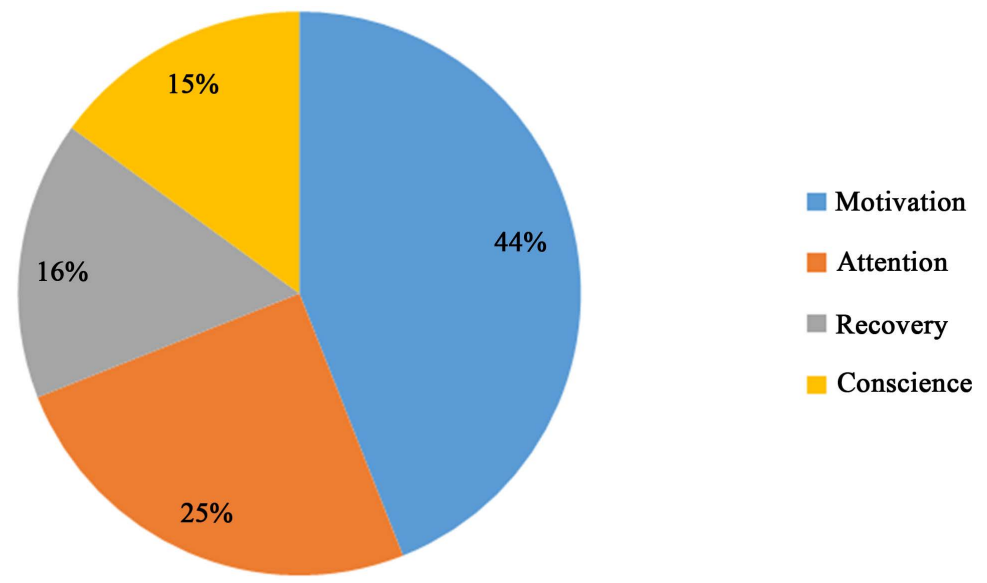

Graph 1. Results of categorization.

manifestations is available at https://goo.gl/CfUCCb. In this topic, an analysis will be made of the category of the greatest number of manifestations, the motivation.

The motivation gained prominence among the four categories of analysis within the Project School of Hackers. This was the auxiliary process of learning that showed the greatest number of manifestations among its subcategories: motives, expectation and intervention. The manifestation that was most perceived among students' actions was the motivation for expectation, that is, when the student shows that he is learning so that his motivation may decrease or increase, depending on the success that he expects of himself if he tries to reach it, or by the success that others expect of him. The number of expectations expressed was 228 perceptions; the one with the smallest numbering was the motivation for intervention, with 57 perceptions, and then, both of them, motivation by motives, with 181 perceptions, as shown in Graph 2 .

An example of perceptions that led to the conclusion of this research can be reflected from the interview with the students, who demonstrated a motivation for "expectation about their own profitability" (Pozo, 2002). One of the students participating in the project said that participating in this project "is more fun than I imagined. It's been six months, but it does not look like it seems like time is just so fast. At first I was very afraid of not knowing things, and I was evolving with time, today I help others. "Another example is in the speech of another student, who said that he liked "a lot the subject, the programming, I really enjoyed learning to program, I did not even know there was this program, Scratch, I thought it was cool to learn." He also heard a demonstration stating that "I hoped it would move more in the system", an activity to which he showed great interest. Even so, he said that "was compensated. At first I thought it was there, I could play on the computer, but then I started to learn and it became fun, so I started to enjoy the course. "The other situations and statements are online in the links of the Analysis Control Table.

It was also possible, through the 1065 total perceptions, to find out that among all the data collected in this research, on the twenty-second of October, 


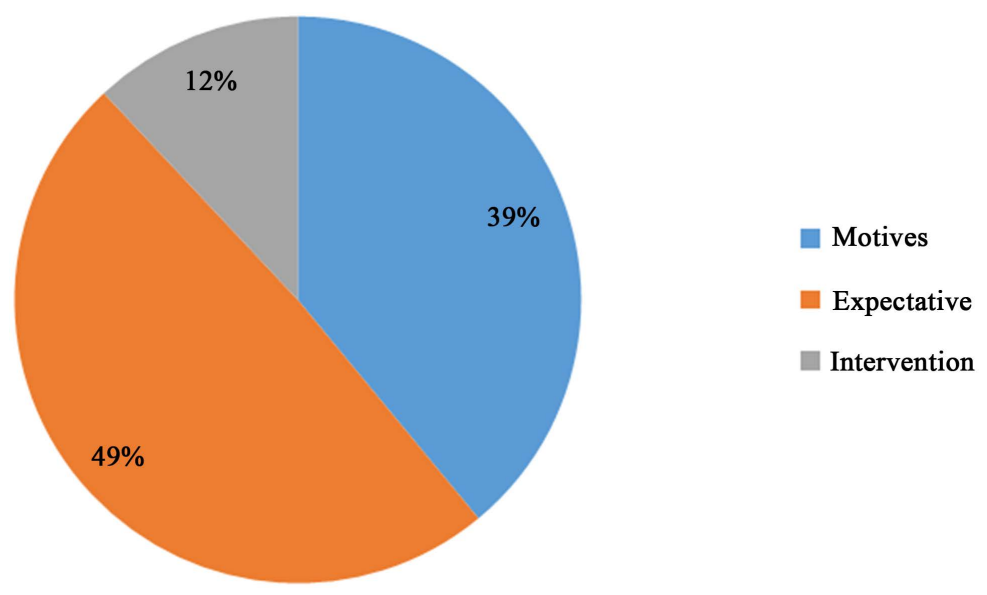

Graph 2. Motivation category.

the four of June and the twenty-seven of August, which are highlighted in Table 2 , the students showed Greater number of manifestations of learning. In this order, a sum of sixty-four, sixty-one and fifty-five manifestations was obtained, which is a higher number than observed in the other workshops observed. From these three workshops in particular, it was possible to understand that students learn and become more motivated when there are moments of more interaction, through dynamics, for example, that they established different classes from the others with the use of Scratch Software and the computer, Each student concentrating on their equipment and schedules. Then, it is concluded that the greater the interaction between apprentices and between apprentices and masters, the terms used by Pozo, the greater is the manifestation of the four auxiliary processes, especially motivation.

We call attention to one last reflection, perceived in a student's speech: "in programming, we are always testing the program, if it does not work, we find a way to correct it, and there in Scratch it shows what is not working" and "we learn more and more, from there we perceive the mistakes". It is important to bring this speech because Is in line with what Papert says when he says that a relevant feature of computer programming is that the child will hardly get it right the first time he programs (Papert, 1985), so it persists and solves the problems until the result is reached It pretends, unlike the model of education in which it exists the right and the wrong, which delays the learning of many children. Thus, everyone learns through their own mistakes or through the mistakes of their peers, so whoever program becomes more tolerant of their mistakes and therefore more motivated. In addition, if the child is not coexisting in an environment where there is only right or wrong, it will become more creative, as it will have to find ways to solve its mistake. In addition, this talk can be related to the strategies suggested by Pozo in which the subject can detect possible errors in their actions, correct them and thus ensure a correct direction of the strategies, guaranteeing success in their actions (Pozo, 2002). Finally, in this case of School of Hackers, it can be affirmed that these positions of Papert and Pozo gain strength when the individual demonstrates motivation, even more if it is motivation by 
expectation, remembering that this category has gained prominence among the four auxiliary processes of the learning of Juan Ignacio Pozo.

\section{Final Considerations}

The research analyzed the broad ramifications of the School of Hackers project on the four auxiliary learning processes of the participating students. The theoretical basis chosen to account for this context, as well as the identification of the categories of analysis, originated from the perspective of Pozo (2002), which deals with the new culture of learning and cognitive learning processes. In this direction, the study sought to analyze how computer programming, a focus of the School of Hackers Project, influences the auxiliary learning processes presented by Juan Ignacio Pozo in his work Apprentice and Master: the new culture of learning, and obtained as a result ends the category of motivation as the most outstanding among the four processes: motivation, attention, recovery and awareness.

After a seven-month field research, it was possible to identify that Notre Dame School students were motivated, either by themselves or by the interference of the monitors, to activate their auxiliary learning processes. At least in some moments, they activated the first mechanism of the attentional system, through the controlled processes, and not only the automated ones; they activated the process of motivation, being it for internal or external reasons, as well as hoped to achieve success in their activities. Also, they recovered and transferred their learning to new contexts, through evocation or recognition, often going through distortions, thinking they remembered, when, in fact, they did not remember their learning. Finally, they became aware of themselves, acting under the three forms of learning consciousness. That is, they used the limited capacity of resources to strategically program their projects through self-knowledge, and finally they applied the three types of control: they planned their tasks, regulated them and evaluated their own outputs and results.

Thus, it can be affirmed that the eleven students, based on their participation in the Project School of Hackers and, in particular, on the occurrences of the twelve manifestations of the four auxiliary processes of learning proposed by Pozo (2002), constructed the knowledge itself about computer programming, rather than simply giving meaning to what they received from the monitors.

We can also point out that, the involvement in challenging tasks and the relativization of the treatment of error not as failure, but as an opportunity-own perception of the act of programming, had greater influence on students' motivation. In this way, they constantly experienced new provocations, which, in this context, stimulated reflection on themselves to become aware of their own cognitive functioning and learning.

In addition, among the observations, three workshops stand out for the considerable amount of perceptions, in comparison to the other workshops. In the analysis, it was observed that all three not only had practical activities, involving programming, through logical blocks, but also played activities involving body 
movement, as well as social interaction between students and monitors. Therefore, the methodology of the other workshops is questioned. Why did other workshops show fewer perceptions?

Lastly, this study can contribute to projects developed at the Notre Dame School, as well as activities carried out in the classroom, in the various teaching disciplines and in all classes, regardless of the age group of the students, as well as influence the next Research Projects, especially those that are connected to the School of Hackers, directing the methodological activities with the Scratch or with other programming software, to the greater control of the student, so that it is constructor of its knowledge without being limited to giving meaning to the one that receives from the monitor.

Thereby, it is believed that students will hardly be as mere receivers of routine learning and will move away from this perception, in which it is still perceived with intensity in many school realities, seen as traditional methodologies. Remembering that the closer the students' learning is to the contexts in which they will be transferred, the easier it will be to recognize them and, the more students transfer their knowledge to new contexts, the easier it will be to recover their learning. Therefore, activities that provide these relationships are very useful and can be worked more intensely in the next workshops.

The research analyzed a broad context of the School of Hackers on the four auxiliary processes of learning of the students participating in the Project, so that later on, this study will continue, tapering each one of the categories or auxiliary processes of learning, as well as its subcategories or forms of manifestations. Therefore, the research enables many forms of future analysis.

\section{References}

Bonilla, M. H. S. (2005). Learner School: Beyond the Information Society. Rio de Janeiro, RJ: Quartet.

Fesakis, G., \& Serafeim, K. (2009). Influence of the Familiarization with “Scratch" on Future Teachers' Opinions and Attitudes about Programming and ICT in Education. Inroads SIGCSE Bulletin, 41, 258-262. https://doi.org/10.1145/1595496.1562957

Papert, S. (1985). Logo: Computers and Education. São Paulo, SP: Brasiliense.

Papert, S. (1994). The Children's Machine: Rethinking the School in the Computer Age. Porto Alegre, RS: Artes Médicas.

Pozo, J. I. (2002). Apprentices and Masters: The New Culture of Learning. Porto Alegre, RS: Artmed.

Pozo, J. I. (2004). The Learning Society and the Challenge of Converting Information into Knowledge. Magazine Pátio, Porto Alegre: Artmed, Year 8, August/October, pp. 34. http://www.udemo.org.br/A\%20sociedade.pdf

Resnick, M. (2007). Learning from Scratch. http://repositorio.ul.pt/bitstream/10451/847/49/20148_ulsd_dep.17852_tm_anexo38a.p df

Rushkoff, D. (2012). The 10 Key Issues of the Digital Age. Schedule Your Future Not to Be Programmed by It. São Paulo: Saraiva. 
Submit or recommend next manuscript to SCIRP and we will provide best service for you:

Accepting pre-submission inquiries through Email, Facebook, LinkedIn, Twitter, etc. A wide selection of journals (inclusive of 9 subjects, more than 200 journals)

Providing 24-hour high-quality service

User-friendly online submission system

Fair and swift peer-review system

Efficient typesetting and proofreading procedure

Display of the result of downloads and visits, as well as the number of cited articles Maximum dissemination of your research work

Submit your manuscript at: http://papersubmission.scirp.org/

Or contact ce@scirp.org 